INVESTIGACIÓN/RESEARCH

\title{
LA DINÁMICA DE GRUPOS PARA EL TRABAJO COOPERATIVO FACILITA LA COMUNICACIÓN
}

Salvador Vidal Raméntol ${ }^{1}$. Universitat Internacional de Catalunya. España. svidal@uic.es

M. Teresa Fuertes Camacho. Universitat Internacional de Catalunya. España. tfuertes@uic.es

\section{RESUMEN}

El objetivo de esta comunicación es resaltar la experiencia llevada a cabo en la UEM, en Enero 2013, en el primer encuentro que se realizo sobre “Diseñando la Educación para una Sociedad Sostenible". Preparamos un taller donde el objetivo era que los profesores aportaran ideas para realizar unos escenarios metodológicos para disminuir nuestra huella ecológica y pudieran implementarlos con sus alumnos. Nuestro objetivo principal era que todos aportaran las experiencias realizadas y surgieran nuevas ideas para ayudar a nuestros alumnos a realizar un consumo más responsable. Nos importaba el que y el cómo. Aplicamos la dinámica de grupo de Panel Integrado, donde cada persona de un grupo tiene que ser portavoz de su equipo, esto implica estar atento y tomar nota de todo lo que dice. El cómo se hacía quería ser una estrategia cognitiva para que en un trabajo en equipo, sobre todo con alumnos, todos fueran protagonistas y así mejorar su competencia comunicativa.

PALABRAS CLAVE. Dinámica de grupo - Trabajo cooperativo - Comunicación Sostenibilidad - Taller.

\footnotetext{
${ }^{1}$ Autor Correspondiente:

Salvador Vidal Raméntol: Profesor Agregado de Didáctica de las Matemáticas en el Grado de EP de la Facultad de Educación. Universitat Internacional de Catalunya. España.

Correo: svidal@uic.es
} 


\title{
THE DYNAMIC GROUPS FOR COOPERATIVE WORK FACILITATES COMMUNICATION
}

\begin{abstract}
The aim of this communication is to highlight the experience carried out in the EMU in January 2013, at the first meeting that took place on "Designing the education for a society sustainable". We prepare a workshop where the objective was that teachers provide ideas to make a methodological scenario to reduce our ecological footprint and could implement them with their students. Our main objective was that all provide the experiences in the past and emerge new ideas to help our students make a more responsible consumption. We mattered which and how. We apply group dynamics Panel integrated, where everyone in a group has to be spokesperson for his team, this means be aware and take note of everything it says. The how was I wanted to be a cognitive strategy for working as a team, especially with students, all were protagonists and thus improve their communicative competence.
\end{abstract}

KEYWORDS: Dynamic group - Cooperative work - Communication - Sustainability - Workshop.

\section{INTRODUCCIÓN}

Cuando nos propusieron de realizar un taller sobre Sostenibilidad nuestro objetivo era sobre todo que fuera muy participativo y todos los asistentes tuvieran la oportunidad de participar. Enseguida pensamos en una Dinámica de Grupo que es la estrategia que aplicamos en muchas de nuestras clases en la Facultad de Educación, Universitat Internacional de Catalunya (UIC). La que nos parecía más adecuada en este contexto fue la del Panel Integrado, donde todos los participantes se convierten en portavoces de su grupo y así evitamos la postura de algunos estudiantes que escuchan en el grupo pero que no toman nota de nada y participan poco. Estas estrategias resultan muy apropiadas para que nuestros alumnos adquieran competencias y demuestren el grado adquirido de las mismas.

\section{METODOLOGÍA}

Si repasamos un poco la teoría, encontramos los orígenes en las características del grupo, tal como lo concibe la dinámica de grupo, son:

1. Un conjunto de dos o más personas identificables por su nombre o tipo.

2. Consciencia de grupo; hay una percepción de unidad, una identificación consciente de unos respecto a los demás.

3. Un mismo sentido de participación en los mismos objetivos.

4. Necesidad de ayudarse mutuamente para conseguir el objetivo para el que se reunieron. 
5. Acción recíproca, todos los miembros se comunican mutuamente.

6. Facilidad para actuar de forma unitaria.

7. Distribución interna de roles que se reconocen entre sí.

Jack R. Gibb², psicólogo americano, elaboró ocho principios básicos que pueden servir de guía y orientación para el aprendizaje del trabajo en grupo:

1. Ambiente. El grupo necesita un entorno físico favorable. Un espacio ni muy grande ni muy pequeño, mesas y sillas que se puedan disponer de tal manera que todo el grupo pueda ver la cara de todos los miembros.

2. Reducción de la intimidación. Las relaciones interpersonales deben ser amables, coordiales y de colaboración. Se reducirán las tensiones para favorecer el trabajo del grupo. Es necesario que las personas se conozcan lo mejor posible y deben ser tolerantes y comprensivas. Se debe poder estar relajado y tranquilo en el grupo.

3. Liderazgo compartido. Todo grupo requiere una conducción que coordine el trabajo, pero en un grupo esta conducción debe ser repartida por todo el grupo para que todos los miembros tengan la posibilidad de desarrollar sus capacidades.

4. Definición del objetivo. Deben definirse con mucha claridad los objetivos del grupo con la participación de todos ya que así aumenta la conciencia colectiva. Es la manera de que el grupo se sienta más unido y trabaje con más interés.

5. Flexibilidad. Los objetivos establecidos se llevarán a cabo; pero, si nuevas necesidades o circunstancias aconsejan una modificación, es necesario que haya flexibilidad y adaptación a los nuevos requerimientos.

6. Consenso. El grupo debe tener una comunicación libre y espontánea, que haga posible un intercambio de opiniones y se pueda llegar a un acuerdo por consenso de todos sus miembros. Esto crea un clima grupal y favorece las relaciones interpersonales y emocionales.

7. Comprensión del proceso. El grupo debe aprender a distinguir entre el contenido de la actividad y la actividad misma, entre lo que se dice y cómo se dice. Hay que estar atento a lo que pasa en el grupo mientras trabaja: los diferentes roles que aparecen, las reacciones, las tensiones, las inhibiciones, los conflictos, etc.

8. Evaluación continua. El grupo debe saber en todo momento si los objetivos y actividades responden a las conveniencias e intereses de los miembros. Para ello hay que hacer una evaluación para saber si el grupo está satisfecho del trabajo que hace.

Estos principios básicos deben irse consiguiendo poco a poco ya que los grupos no nacen sino que se van haciendo poco a poco.

2 Gibb,J.R. (1982): Manual de dinámica de grupo. Buenos Aires. Humanitas. 


\section{ANÁLISIS Y DISCUSIÓN}

Las técnicas de grupo son los procedimientos o la manera de organizar y desarrollar la actividad, teniendo en cuenta los conocimientos que proporciona la Dinámica de Grupos, y suficientemente probados en la experiencia. Esta experiencia es la que permite afirmar, según Thelen, que una técnica adecuada tiene el poder de activar los impulsos y las motivaciones individuales y de estimular tanto la dinámica interna como la externa.

Las técnicas de grupo son instrumentos para conseguir el objetivo del grupo. Dice Thelen Una técnica no es por sí misma ni buena ni mala, pero puede ser aplicada eficazmente o desastrosamente.

Para seleccionar la técnica más conveniente se debe tener en cuenta una serie de factores:

1. Según los objetivos que se persiguen. Hay diferentes estructuras de dinámica de grupos según los objetivos que se persiguen. Hay técnicas especiales para promover el intercambio de ideas, otras para tomar decisiones, otras favorecen el aprendizaje de conocimientos, otras facilitan la comprensión de diversas situaciones, otras promueven la participación total, otras para desarrollar el pensamiento creador, otras para promover las actitudes positivas o la capacidad de análisis, etc. Por lo tanto hay que clarificar el objetivo que se persigue.

2. Según la madurez y entrenamiento del grupo. Algunas son fácilmente aceptadas por el grupo y otras provocan al principio ciertas resistencias por su novedad o por ser externas al grupo. Para grupos nuevos, no experimentados en la actividad grupal, convendría seleccionar aquellas técnicas más sencillas y más afines a los miembros del grupo. Así pues, teniendo en cuenta que nuestros alumnos en general están más predispuestos en una reunión a asistir escuchando que a actuar, deberíamos empezar por técnicas que impliquen poca participación activa de los miembros, y poco a poco ir evolucionando a técnicas de más participación, a medida que el grupo se hace más maduro.

3. Según el número de miembros del grupo. El comportamiento de los grupos depende muchas veces del número de los componentes. En grupos pequeños se da una mayor cohesión e interacción, existe más seguridad y confianza, las relaciones son más estrechas y amistosas y se llega más fácilmente al consenso. En estos grupos podrán utilizarse con éxito técnicas como debates dirigidos, pequeños grupos de discusión, estudio de casos...

4. En los grupos grandes se dan las características opuestas. Habría que dividir estos grupos en subgrupos. La conducción de un grupo grande exige una mayor experiencia por parte del conductor. Hay que empezar por técnicas más apropiadas a este tipo de grupo como el simposio, panel integrado, Phillips 66, seminario...

5. Según el ambiente físico. Cuando escogemos una técnica hay que tener presente la realidad de espacio y de tiempo. Hay técnicas que requieren una sala amplia 
y las hay que exigen más tiempo en su realización. Estas dos condiciones deben tenerse presentes ya que el grupo actúa coaccionado bajo la presión de tiempo y de espacio. También hay que tener en cuenta si se necesitan herramientas auxiliares, como pizarra, láminas, retroproyector, pantalla, ordenador... La disponibilidad de los elementos condiciona también la selección de la técnica.

6. Según las características del medio externo. Hay unas circunstancias externas al grupo, clima afectivo de carácter psicológico. Cualquier innovación que rompa la rutina del centro necesita la aprobación o aceptación de la institución donde se lleva a cabo la dinámica de grupos. Una vez conseguido el clima de aceptación, es conveniente, para empezar, escoger aquellas técnicas que más se asemejan al tipo de actividad que ya se realiza en el centro. También habrá que tener presente el tipo de objetivo que se proponga en las primeras dinámicas de grupo. Aceptan con mucha facilidad las destinadas a la acción educativa para mejorar el aprendizaje.

7. Según las características de los miembros. Los grupos varían en función de los componentes: edad, interés, nivel de conocimientos, expectativas, predisposición, experiencias anteriores... Por tanto, según lo que tengamos delante tendremos que aplicar una técnica $u$ otra.

8. Según la experiencia del conductor de la reunión. Hay que tener presente si es una técnica sencilla o complicada y si intervienen grupos pequeños o grandes grupos. Hay que conocer bien las posibilidades ya que, según la técnica, se puede producir tensión. Hay que coger confianza y seguridad para ir ampliando las diferentes técnicas. Con la experiencia se va viendo que hay técnicas que deben adaptarse al grupo. Lo que es muy importante es que la técnica sea útil y consiga los objetivos propuestos para el grupo.

\subsection{Algunas normas generales.}

Cada técnica tiene sus normas según lo que se pretende conseguir, pero hay una serie de normas que son de carácter general, que salen de la misma Dinámica de Grupo y que hay que tener presentes ${ }^{3}$ :

1. Antes de aplicar técnicas de dinámica de grupo es necesario conocer sus fundamentos teóricos.

2. Antes de aplicar una técnica concreta es necesario conocer su estructura, la dinámica, las posibilidades y los riesgos.

3. Hay que seguir al máximo el procedimiento señalado en cada técnica.

4. Al aplicar una técnica hay que tener muy claro el objetivo que nos proponemos con el grupo en cuestión.

5. Para aplicar una técnica es necesario que haya un clima cordial y afable.

6. En todo momento debe haber un clima de colaboración y cooperación.

7. Es necesario que todos los componentes tengan una participación activa.

${ }^{3}$ Vidal, S. (2009): Estrategias para la enseñanza de las Matemáticas en Secundaria. Barcelona. Laertes. 
8. Hay que tener conciencia de grupo. El grupo existe gracias a cada uno de los componentes.

9. Para participar en un grupo hay que tener buena intención y ganas de trabajar.

10. Todas las técnicas de dinámica de grupo tienen como finalidad:
a. Desarrollar el sentimiento de nosotros.
b. Enseñar a pensar de forma activa.
c. Enseñar a escuchar de forma comprensiva.
d. Desarrollar capacidades de cooperación, responsabilidad, autonomía, creatividad, intercambio de opiniones, colaboración.
e. Superar tensiones, vencer falsos temores y crear sentimientos de seguridad.
f. Crear una actitud positiva ante los problemas de las relaciones humanas y crear un clima favorable a la adaptación social del individuo.

\subsection{Actitud del alumno ante las técnicas de dinámica de grupo.}

Es necesario un cambio de actitudes por parte de los alumnos a la hora de trabajar en grupo:

\begin{tabular}{|ll|}
\hline ACTITUD TRADICIONAL & ACTITUD COMPETENCIAL \\
\hline - cambiar escuchar & por hablar \\
- cambiar las normas rígidas & por libertad y autonomía \\
- cambiar las coacciones & por responsabilidad \\
- cambiar la intimidación & por cooperación \\
- cambiar la actitud defensiva & por permisividad \\
- cambiar las sorpresas & por seguridad \\
- cambiar el profesor centralista & por planificación colectiva \\
- cambiar el autoritarismo del profesor & por el consenso del grupo \\
- cambiar la evaluación del profesor & por la autoevaluación del grupo \\
\hline
\end{tabular}

Como dice Gibb Los grupos no nacen sino que se hacen.

Hay que evitar las actitudes defensivas y hay que crear un clima agradable y confiado, donde todos los miembros se sientan cómodos y aceptados por todos. 


\subsection{El conductor del grupo.}

Hay que tener presentes dos aspectos:

1. Es necesario conocer los fundamentos teóricos y los principios básicos de las técnicas de dinámica de grupo y sus procedimientos.

2. Hay que modificar, hacia una mayor seguridad, las actitudes y conductas profesionales.

El primer aspecto no tiene problema ya que se trata de un aprendizaje intelectual sin ningún secreto; el segundo es más delicado ya que hay que cambiar conductas y actitudes muy arraigadas a lo largo de los años de la experiencia docente. En resumen, hay que hacer un cambio de rol.

\begin{tabular}{|ll|}
\hline ROL DOCENTE TRADICIONAL & ROL DE CONDUCTOR DE GRUPO \\
Tiene todo el saber & Promueve el saber y enseña a aprender \\
Tiene autoridad & Promueve responsabilidad \\
Toma decisiones & Enseña a tomar decisiones \\
Hace que lo escuchen & Escucha y hace hablar \\
Aplica normas & Utiliza técnicas de grupo \\
Marca los objetivos & Propone objetivos \\
Se preocupa de la disciplina & Se preocupa del proceso grupal \\
Evalúa al alumno & Evalúa con el alumno \\
Trabaja con alumnos & Trabaja con el grupo \\
Controla, sanciona & Estimula, orienta, tranquiliza \\
\hline
\end{tabular}

Un buen conductor del grupo es aquél que ayuda a funcionar. Tiene un rol de líder o de animador distinto del que podemos encontrar a veces.

Podemos hallar diferentes tipos de líderes dentro de los grupos: 
- Líder autoritario: Toma las decisiones, da órdenes y normas, impone su criterio, es el único responsable, da pocas explicaciones de sus acciones o ninguna, señala los objetivos, distribuye el trabajo a los componentes del grupo. Los grupos dirigidos autocráticamente tienen más hostilidad, descontento, competición: sus miembros son poco creadores y más dependientes. Las reacciones pueden ser agresivas en unos y apáticas en otros.

- Líder paternalista: Es amable, cordial, paternal, deja discutir, pero al final expone sus razones de peso que deben ser aceptadas por el bien de todos, es el protector y el experto. No acepta ninguna observación o que se le discuta su decisión. En estos grupos, los componentes no se desarrollan, no crecen porque no tienen la oportunidad de tomar decisiones y aprender de sus propios errores. No adquieren responsabilidad. - La inmadurez genera paternalismo y el paternalismo genera inmadurez (Gibbs).

- Líder democrático: Confía en la capacidad del grupo, favorece las discusiones, pide y tiene en cuenta las opiniones del grupo antes de tomar ninguna decisió. La responsabilidad es compartida por el grupo. Da explicaciones y acepta críticas. Da libertad para trabajar a los miembros del grupo. Los grupos de estructura democrática están más motivados para trabajar. Las relaciones personales son más estrechas y cordiales. Hay más comunicación, menos hostilidad y mayor desarrollo personal del grupo.

- Líder pasivo (laissez-faire): Actúa poco, deja hacer a los miembros, no toma decisiones ni orienta. Las discusiones son incontroladas. La estructura del grupo es débil y de carácter individualista. El grupo no tiene incentivos, se discute por discutir sin llegar a tomar decisiones. Hay poco espíritu cooperador y los componentes se desarrollan poco.

- Liderazgo participativo: Es el grupo que actúa por consenso, todos los componentes trabajan en conjunto para lograr una buena cohesión de grupo. Lo que importa es el crecimiento y desarrollo de todos sus miembros, ninguno de los cuales es líder. Todos los objetivos y actividades son escogidos por grupo. Esta estructura puede funcionar en grupos suficientemente maduros y experimentados. Es la meta para todos los grupos.

Para aplicar técnicas de dinámica de grupo es necesario un estilo de liderazgo democrático ya que es el que más se ajusta a los fundamentos teóricos y filosóficos de la dinámica de grupo: desarrolla la personalidad, responsabilidad, iniciativa propia, creatividad, autonomía, cooperación, solidaridad, colaboración...

\subsection{Sugerencias que se deducen del marco teórico consultado.}

1. Hay que cambiar la postura de profesor tradicional. Si quieres que te escuchen di cosas interesantes para el grupo. Hazlos participar de forma activa en 
proyectos y decisiones. Hay que ser aceptado por el grupo; tú no te puedes imponer.

2. No amenaces ni castigues, intenta ser cordial y comprensivo. Analiza las situaciones difíciles, empezando por analizar tu propia conducta. No presiones ni directa ni indirectamente al grupo para que acepte tu punto de vista.

3. Ten confianza en el grupo, la juventud es capaz de desarrollar cosas muy valiosas a poco que les dejemos ser ellos mismos.

4. Pon buena voluntad en aceptar aquellas decisiones que tome el grupo.

5. Estimula la comunicación horizontal en todo el grupo. La interacción no sólo debe ser contigo sino entre ellos. Acostúmbrate a saber escuchar.

6. Ten en cuenta los sentimientos, son muy importantes en las relaciones humanas.

7. Hay que dar muestras de seguridad, para ello hay que tener muy bien preparada la técnica que utilizaremos.

8. Sigue el procedimiento de cada técnica, pero piensa que no hay ningún grupo igual y hay que tener unas dosis de ingenio y creatividad.

\section{CONCLUSIONES}

Realizamos una primera parte más teórica de exposición de diferentes escenarios metodológicos empleados en la educación superior para la implementación curricular de competencias en sostenibilidad. En la segunda parte se llevo a cabo un trabajo en equipo. Dividimos los asistentes, 12 personas, en tres grupos, grupo 1, grupo 2 y grupo 3, de cuatro personas cada uno, y cada grupo debía pensar distintos escenarios metodológicos para llevar a cabo las distintas competencias propuestas. Todos los asistentes disponían de unas fotocopias con la lista de las competencias que teníamos que trabajar. Se pedía a cada grupo que por lo menos aportaran cuatro escenarios, uno cada uno.

Cada grupo fue trabajando de forma autónoma y aportando ideas de lo que ya están haciendo y de nuevos escenarios que aparecían en el trabajo en grupo.

Después de 45 minutos, se dijo a los distintos grupos que tomaran nota de todo lo que se había dicho porque debían compartirlo con los distintos compañeros de los otros grupos, de forma que todos pasaban a ser portavoces de su grupo. Utilizamos una técnica de dinámica de grupos, que utilizamos en el aula para que todos los alumnos se sientan responsables del trabajo realizado en equipo. La técnica se llama "panel integral" y es muy útil para realizar un trabajo cooperativo. Consiste en hacer grupos, en nuestro caso tres grupos de cuatro personas, y cada persona del grupo tiene una letra. 


\begin{tabular}{|c|c|c|c|c|}
\hline 1 & $\mathrm{~A}_{1}$ & $\mathrm{~B}_{1}$ & $\mathrm{C}_{1}$ & $\mathrm{D}_{1}$ \\
\hline 2 & $\mathrm{~A}_{2}$ & $\mathrm{~B}_{2}$ & $\mathrm{C}_{2}$ & $\mathrm{D}_{2}$ \\
\hline 3 & $\mathrm{~A}_{3}$ & $\mathrm{~B}_{3}$ & $\mathrm{C}_{3}$ & $\mathrm{D}_{3}$ \\
\hline
\end{tabular}

Tabla 1. Organización de la Dinámica de Grupo

Una vez cada grupo ha llegado a sus conclusiones, se hacen nuevos grupos con un representante de cada grupo, en nuestro caso se hicieron cuatro grupos de tres personas, el grupo de las A, de las B de las C y de las D, de forma que todos pertenecían a grupos distintos y debían compartir con los nuevos compañeros el trabajo realizado en su grupo. Si tomaban nota, tendrían toda la información de todos los grupos.

Una vez realizado el intercambio de información se recogieron las aportaciones de los distintos grupos y se redactaron las conclusiones del taller:

- Trabajo en red, crear un "ovillo en red".

- Celebrar efemérides. Actividades y juegos relacionados con la sostenibilidad.

- Formación del voluntariado. Se insistió en la necesidad de implementar el "Aprendizaje servicio" que ya realizan algunas universidades y está dando un muy buen resultado.

- Trabajo en equipo. Se vio la necesidad de introducir dinámicas de grupo que favorecen la cooperación de los alumnos.

- Trabajar con la diversidad de información. Saber organizarla.

- Realizar proyectos entre diferentes materias. La UDC presento un proyecto de la Facultad de Educación donde trabajan conjuntamente con las asignaturas de Lenguaje, Matemáticas, Sociales y Ciencias Experimentales. Para fomentar la participación, el mejor proyecto tiene un premio de 1000 fotocopias gratis.

- Resolución de casos (ABP) con dilema ético en las asignaturas de Finanzas.

- Buscar y comentar la "Eco noticia". Buscar noticias relacionadas con el consumo responsable y comentarla en clase.

- Consumo responsable, estudio arquitectónico de edificios para ser más sostenibles.

- “Refléjate - refléjame”. Reflejar la realidad social y representarla.

- Publicidad ética. Se crea el propio anuncio con códigos éticos y se hace la reflexión. 
Al final conseguimos el objetivo previsto ya que las doce personas que participaron del taller tuvieron que intervenir $y$ todos conseguimos nuevos escenarios metodológicos para implementar en nuestra Universidad competencias de Sostenibilidad. La dinámica de grupos facilito la comunicación y una vez más, la práctica nos convence de la utilidad del trabajo cooperativo.

\section{BIBLIOGRAFÍA}

Abad, M. y Benito, M.L. (2006): Cómo enseñar juntos a alumnos diferentes. Aprendizaje cooperativo. Zaragoza. Egido.

Gibb, J.R. (1982): Manual de dinámica de grupo, Buenos Aires. Humanitas.

Klaus, A. (1990): Práctica de la dinámica de grupos. Barcelona. Herder.

Leal, W. (2012): Sustainable Development at Universities: New Horizons. Peter Lang Scientific Publishers.

Lozano, R. (2006): Envisioning Sustainability Three-Dimensionally. Greening of Industry Network: Cardiff.

Pujolas, P. (2008): Nueve ideas clave. El aprendizaje cooperativo. Barcelona. Grao.

Rees, W. E. (2002): “An ecological economics perspective on sustainability and prospects for ending poverty". Population and Environment 24(1): 15-46.

Thelen, E. (1994): “Three-month-old infants can learn task-specific patterns of interlimb coordination". Psychological Science, 5, p. 280-285.

Vidal, S. (2011): «Good morning, numbers day». Australian Primary Mathematics Classroom. (The Australian Association of Mathematics Teachers Inc). 16 (3), p. 25-28.

Vidal, S. (2009): Estrategia para la enseñanza de las matemáticas en secundaria. Barcelona. Laertes.

Vidal, S. (2005): Dia del número, motivació de la matemàtica. Barcelona. Publicacions de l'Abadia de Montserrat.

Vidal, S.; Fuertes, M.T. i altres (2008): Adaptació dels estudis de Magisteri a l'EEES: el cas de la UIC. Barcelona.Prohom Edicions.

Vidal, S. (2010): «La comunicación en la didáctica de las matemáticas». Revista Vivat Academia (Universidad Complutense de Madrid). No 112, p. 1-9. 
Vidal, S. (2011): «La situació de la didàctica de les matemàtiques a la secundària catalana. Anàlisi de l'estat de l'ensenyament i l'aprenentatge». Temps d'Educació, (Universitat de Barcelona), 41, p. 185-199.

Vidal, S. (2013): Día del número, motivación de la matemática. Publicia.

\section{Salvador Vidal Raméntol}

Licenciado en Ciencias, UB. Doctor en Ciencias de la Educación por UB. Miembro del Claustro de Doctores de la UB. Miembro del grupo de Investigación SiRSU sobre Sostenibilidad y responsabilidad Social Universitaria de la UIC. Profesor del Máster Universitario en Investigación en Ciencias Humanas, Sociales y Jurídicas. Miembro de la Escuela de Doctorado, comisión de Calidad. Vicedecano de la Facultat de Educación (2003-2010). Miembro de honor del CSEDOHC Consejo Superior Europeo e Iberoamericano de Doctores y doctores Honoris Causa.

\section{Teresa Fuertes Camacho}

Doctora por la UIC. Máster en Ciencias Humanas, Sociales y Jurídicas (UIC), Licenciada en Psicopedagogía UOC y diplomada en Magisterio URL. Especialista en asesoramiento curricular. Profesora adjunta de la Universitat Internacional de Catalunya (UIC). Responsable del Prácticum de Educación. Vocal de la CADU (Comité Evaluación Docencia Universitaria- 2011). Miembro de la Red Universitaria de Aprendizaje-Servicio y Responsabilidad Social Universitaria. Miembro del Grupo de Investigación sobre profesionalización (UIC). 